\title{
Observaciones sobre la larva del cangrejo marino tropical (Decapoda: Porcellanidae) Petrolisthes armatus en el Golfo de Nicoya, Costa Rica
}

\author{
Edgardo Díaz-Ferguson ${ }^{1}$, Daisy Arroyo $^{2}$, Alvaro Morales ${ }^{2}$ \& José A. Vargas ${ }^{2}$ \\ 1. Department of Zoology, University of Florida. PO Box 118525, Gainesville, FL 32611; edgard03@ufl.edu \\ 2. Centro de Investigación en Ciencias del Mar y Limnología (CIMAR), Universidad de Costa Rica, 2060 San José, \\ Costa Rica. Fax (506) 2207-3280
}

Recibido 19-II-2007. Corregido 30-VI-2008. Aceptado 31-VII-2008.

\begin{abstract}
Observations on the larvae of the tropical marine crab Petrolisthes armatus in the Gulf of Nicoya, Costa Rica. During October and November 1998 (rainy season), and December, February and March 1999 (dry season), larvae distribution of Petrolisthes armatus and associated decapods were sampled in three different sites at the Punta Morales peninsula, Gulf of Nicoya, Costa Rica. A total of 6014 decapod larvae were collected, and 73 belonged to the genus Petrolisthes spp. No significant total larval density differences were found between seasons ( $>0.05$ ); but there was a greater density of $P$. armatus and other decapod larvae $(\mathrm{p}>0.05)$ during the dry season. In addition, no significant variations on temperature and oxygen concentration were observed. P. armatus larval abundance was higher during low tide, in contrast with other decapod larvae. Significant differences among sites were found for other decapod larvae, but not for P. armatus. The only parameter that varied significantly between seasons was salinity and results demonstrate that this factor regulates temporal concentration of larvae. Moreover, flow-tide oscillations were the most important spatial factor in larval dynamics. We propose this mechanism: P. armatus liberates larvae during high tide; these larvae leave the coastal area during low tide and return to the rocky intertidal ecosystem during high tide, when they are ready to settle as megalopa (pre-juvenile stage). Parallel laboratory observations showed higher survival rates at lower salinities (15 ups against 35 ups) and that the duration of the period from zoea I to megalopa was, in average, 19 days. Rev. Biol. Trop. 56 (3): 1209-1223. Epub 2008 September 30.
\end{abstract}

Key words: Crustacea, Porcellanidae, Petrolisthes armatus, larvae, population dynamics, Gulf of Nicoya, Costa Rica.

La mayoría de los invertebrados marinos, muestran ciclos de vida que incluyen estadios pelágicos iniciales, con su fase juvenil y adulta béntica (Ekman 1996, McEdward 1997). En las zonas tropicales este tipo de ciclo de vida se observa en el $80 \%$ de los invertebrados marinos (McEdward 1997). Los mecanismos de dispersión, retención y asentamiento larval de una especie, son en cierta forma estrategias evolutivas que han permitido a las poblaciones de adultos garantizar su éxito en un determinado hábitat (Olson 1985). Sin embargo, el estudio del estadio pelágico se ha limitado a especies de interés comercial o se ha estudiado aisladamente los factores que afectan a las larvas en el plancton, sin considerar la dinámica de la población de adultos (Watzing 1986).

Para un mejor entendimiento de la dinámica poblacional de los decápodos, es necesario integrar procesos y factores que ocurren en ambos componentes del medio marino (planton y bentos). Gran parte de las especies del Orden Decapoda presentan etapas de transición entre los componentes bénticos y pelágicos del medio (Sastry 1983, McEdward 1995). A pesar de este hecho, pocos estudios han hecho énfasis en la importancia del asentamiento, selección del hábitat, dispersión de las postlarvas y aporte de larvas en la dinámica poblacional de invertebrados marinos (Watzin 1986, Jensen 1991, 
McConnaughey et al. 1992, McConnaughey et al. 1995, Miron et al. 1995, Osman y Whitlatch 1995, Eckman 1996).

La especie Petrolisthes armatus es un cangrejo anomuro de la familia Porcelanidae que habita el litoral rocoso intermareal en las costas de Africa y América, en las que se ha planteado que es dominante y que su variación poblacional regula otras poblaciones de decápodos que habitan este hábitat (Gore 1972, Oliveira y Masunari 1995). Recientemente se ha reportado extensión en su ámbito de distribución hasta las costas de Sur Carolina, Estados Unidos, lo que demuestra el potencial como especie colonizadora de $P$. armatus (Ray 2005). Para esta especie al igual que para otros anomuros, los estudios se limitan a la descripción de aspectos de la embriología y distribución de la larva en zonas específicas. El género Petrolisthes ha sido identificado en los inventarios de decápodos realizados en esta zona y en cuanto a su transporte larval se ha planteado que existe una exportación de la larva por superficie a la zona externa del estero en estadios iniciales y posteriormente una reincorporación a la zona costera durante las fases finales del desarrollo pelágico (Dittel y Epifanio 1982, Dittel et al. 1991). Además, en la zona del Golfo de Nicoya no existen estudios sobre la distribución de la larva en el plancton y los factores que regulan su densidad en la columna de agua. No obstante, para los decápodos en general debido a su importancia comercial y a la abundancia del grupo en la zona, existen estudios que relacionan la distribución de postlarvas de peneidos con poblaciones adultos, así como para braquiuros cuya existencia adulta es parcialmente pelágica (Dittel et al. 1985, Palacios et al. 1993, Kitami et al. 1997).

El presente trabajo de investigación estudia la distribución espacial y temporal de las larvas del género Petrolisthes así como los factores que regulan la densidad de las larvas de esta especie, confirmando los mecanismos de dispersión y distribución planteados para las prezoeas y zoeas de este género. De esta forma se podrá establecer la relación entre la fluctuación de las características poblacionales en fase pelágica con la variación de algunas de las características poblacionales de la especie en su fase adulta. Así mismo, se trata de integrar los aspectos del comportamiento ligados al desarrollo y duración de los estadios larvales, hasta la fase de megalopa; con lo cual se tendrá una aproximación más exacta de la duración del desarrollo pelágico y un marco de referencia para futuros estudios sobre reclutamiento y etología de la especie. El estudio de la abundancia de $P$. armatus en zonas rocosas en el Golfo de Nicoya ha sido realizado por Díaz-Ferguson y Vargas (2002), en este trabajo complementario aportamos datos sobre las formas larvales.

\section{MATERIALES Y MÉTODOS}

Area de estudio: Los muestreos de plancton se realizaron en tres sitios cercanos a la costa en la zona interna del Golfo de Nicoya; costa Pacífica de Costa Rica (Fig. 1). El sitio 1 se ubicó a $10^{\circ} 03^{\prime} 44 \mathrm{~N}$ y $84^{\circ} 57^{\prime} 34 \mathrm{~W}$ aproximadamente a 50 metros del litoral rocoso de la península de Punta Morales. El sitio 2 se ubicó a $10^{\circ} 03^{\prime} 21^{\prime \prime} \mathrm{N}$ y $84^{\circ} 57^{\prime} 32^{\prime \prime} \mathrm{W}$, aproxi-

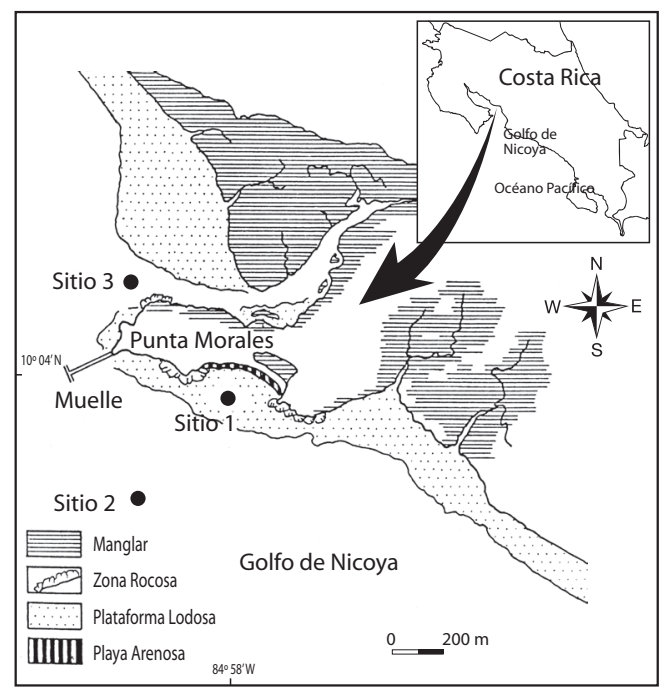

Fig. 1. Sitios de recolecta de muestras de plancton en la zona de Punta Morales, Golfo de Nicoya, Costa Rica. Modificado de La Cruz \& Vargas, Rev. Biol. Trop. 35" 367. 1987. 
madamente a 600 metros de la costa. El sitio 3 se ubicó a $10^{\circ} 04^{\prime} 11^{\prime \prime} \mathrm{N}$ y $84^{\circ} 57^{\prime} 59^{\prime \prime}$ W en la zona interna del estero de Punta Morales. En esta área la temperatura del agua oscila entre los $20{ }^{\circ} \mathrm{C}$ y $31{ }^{\circ} \mathrm{C}$ con media anual de $29^{\circ} \mathrm{C}$, mientras que la precipitación alcanza sus máximos durante octubre $(705 \mathrm{~mm})$ y los mínimos en febrero y marzo.

Recolecta de larvas en el plancton: En meses representativos de cada estación (seca y lluviosa), se muestreó con una red de plancton de $280 \mu \mathrm{m}$, con diámetro de boca de 0.5 m. En cada uno de los sitios se realizaron 2 arrastres, uno superficial y otro a tres metros de profundidad. La duración de los arrastres fue de 3 minutos a una velocidad de un nudo. El volumen de agua filtrado se determinó a través de un medidor de flujo que se ajustó a la boca de la red. Posterior a la recolecta, las muestras se fijaron con formalina al 5\%. Una vez en el laboratorio las muestras fueron lavadas con agua dulce, colocadas en alcohol al $70 \%$, fraccionadas hasta $1 / 16$ con el separador Folson y luego cuantificadas por medio de un contador manual en la cámara Bogorov. Las larvas de Petrolisthes sp. fueron separadas del total de las larvas de decápodos recolectadas. Para la identificación de los individuos del género Petrolisthes sp. se utilizó la descripción realizada por Gore (1972).

Parámetros físico-químicos: Los parámetros físico-químicos de temperatura, penetración de luz, salinidad y oxígeno disuelto se determinaron en cada uno de los sitios y profundidades de muestreo. Para la medición de la temperatura y oxígeno disuelto se utilizó un sensor polarográfico. Para la obtención de muestras de agua a profundidad de tres metros se utilizó una botella nisken dentro de la cual una vez en la embarcación se introdujo el sensor para obtener los valores de temperatura y oxígeno. La salinidad se determinó a la profundidad de tres metros con un refractómetro óptico con compensación de temperatura. La penetración de luz en la columna de agua se determinó mediante un disco de secchi.
Desarrollo larval en el laboratorio: En la zona de estudio se recolectaron hembras ovígeras cuyas características de tamaño y nivel de maduración de los huevos fueran similares. A estos individuos se les colocó un recipiente a $25{ }^{\circ} \mathrm{C}$ para su transporte al laboratorio. En el laboratorio se les llevó gradualmente hasta la temperatura de recolecta, $29{ }^{\circ} \mathrm{C}$. Las hembras ovígeras se transfirieron a acuarios donde se les dio un baño que consistió en 3 gotas de formol en $500 \mathrm{~mL}$ de agua de mar filtrada hasta 0.5 micras por tres minutos. Una vez lavadas las hembras, estas se pasaron a acuarios individuales de $0.5 \mathrm{~mL}$. El agua que se utilizó en estos acuarios fue filtrada a través de mallas de $10,5 \mathrm{y}$ $2 \mu \mathrm{m}$ y posteriomente esterilizada con radiación ultravioleta. En estos acuarios la aereación fue continua pero de baja intensidad. La salinidad se mantuvo en un ámbito de 30 a 35 ups y la temperatura a $27^{\circ} \mathrm{C}$. Una vez liberadas las larvas se elevó la temperatura hasta un ámbito aproximado de $27.5-28{ }^{\circ} \mathrm{C}$. La alimentación de las larvas consistió en nauplius de Artemia salina $(10 \mathrm{~mL})$, el rotífero Brachionus sp. (10 mL) y células de la microalga Chaetoceros sp. (60 mL). A partir del octavo día, se varió la cantidad de alimento, disminuyendo la cantidad de fitoplancton y proporcionando mayor cantidad de Artemia y rotíferos. Diariamente se registraron las características de las larvas tanto de desarrollo como de comportamiento. También se cuantificó la densidad total de las larvas por acuario. Para estudiar el efecto de la salinidad se compararon cuatro repeticiones para la salinidad máxima y mínima; cuyos valores se basaron en los máximos y mínimos obtenidos en el medio natural (15 y 32 ups). Una vez liberadas las larvas; se mantuvieron a una temperatura de $30^{\circ} \mathrm{C}$ y luego se distribuyeron en ocho acuarios, utilizando cuatro de estos para cada salinidad. Diariamente se contó el número de larvas y se estimó el porcentaje de supervivencia; también se cuantificó el total que alcanzó el estadio de zoea II para la salinidad en cuestión.

Métodos estadísticos: Para el estudio de la variación de la densidad de larvas de porcelánidos se realizaron pruebas de estadística 
no paramétrica. Para observar la variación entre profundidades y mareas se utilizó la prueba de U-Mann Withney, mientras que para la variación de la densidad por sitio se realizó una prueba de Kruskal Wallis. Para el estudio de las variaciones en el total de las larvas de decápodos espacial y temporalmente se utilizaron pruebas de estadística paramétrica (ANDEVA de una vía) debido al número de individuos y distribución de los datos. También se tomaron en cuenta valores de estadística descriptiva de la población muestreada (media, desviación estandard y varianza). Para comparar la variación estacional de la densidad de larvas de porcelánidos se utilizaron valores de densidad total por estación y se les aplicó a los mismos una prueba de U-Mann Withney. Esta misma prueba se utilizó para ver el efecto de salinidad así como para comparar la variación espacio-temporal de los parámetros fisico-químicos.

\section{RESULTADOS}

Variación espacial y temporal de los parámetros físico-químicos de la columna de agua: Los máximos, mínimos y desviaciones de la temperatura por estación y profundidad se muestran en el Cuadro $\mathrm{N}^{\mathrm{o}} 1$ y 2, Fig. 2a y b. No se encontró variación significativa de este parámetro por estación. Los valores superficiales fueron cercanos o menores de los $30^{\circ} \mathrm{C}$. En cuanto a los valores de temperatura a $(3 \mathrm{~m})$, esta se mantuvo prácticamente constante entre las fechas muestreadas con valores inferiores a los $30^{\circ} \mathrm{C}$. Entre profundidades se observaron diferencias significativas $(\mathrm{p}<0.05)$ para este parámetro (Cuadro 1). Entre sitios y mareas no se observaron diferencias significativas para este parámetro $\mathrm{p}>0.05$.

Para la salinidad si se observó diferencias significativas al analizarse los datos de forma estacional $(\mathrm{p}=0.003)$. Para ambas profundidades (superficial y profunda), los mínimos de salinidad se registraron en la segunda fecha de muestreo correspondiente al mes de noviembre (Fig. 3) mientras que los máximos se registraron a partir d diciembre en la estación seca. Espacialmente la salinidad entre sitios, profundidades y mareas no varió significativamente ( $p>0.05)$ (Cuadro 1).

Otros parámetros estudiados fueron el oxígeno disuelto y la penetración de luz los cuales

CUADRO 1

Valores estadísticos de la variación estacional de la salinidad, oxígeno y temperatura

TABLE 1

Statistical values of seasonal variation of salinity, oxygen, and temperature

\begin{tabular}{|c|c|c|c|c|}
\hline & Parámetros estadísticos & $\begin{array}{l}\text { Salinidad } \\
\text { ppt }\end{array}$ & $\begin{array}{l}\text { Oxígeno } \\
\mathrm{mg} / \mathrm{ml}\end{array}$ & $\begin{array}{c}\text { Temperatura } \\
{ }^{\circ} \mathrm{C}\end{array}$ \\
\hline \multirow{5}{*}{ Estación seca } & Mínimo & 20 & 3.10 & 25 \\
\hline & Máximo & 32 & 7.8 & 32 \\
\hline & Media & 27 & 5.08 & 27 \\
\hline & Varianza & 7.1 & 1.43 & 2.2 \\
\hline & Desviación Estandard & 2.67 & 1.19 & 1.49 \\
\hline \multicolumn{5}{|c|}{ Estación lluviosa } \\
\hline & Mínimo & 15 & 2.9 & 26 \\
\hline & Máximo & 30 & 7.8 & 30 \\
\hline & Media & 25 & 4.9 & 28 \\
\hline & Varianza & 13 & 2.0 & 1.31 \\
\hline & Desviación Estandard & 3.7 & 1.41 & 1.14 \\
\hline
\end{tabular}



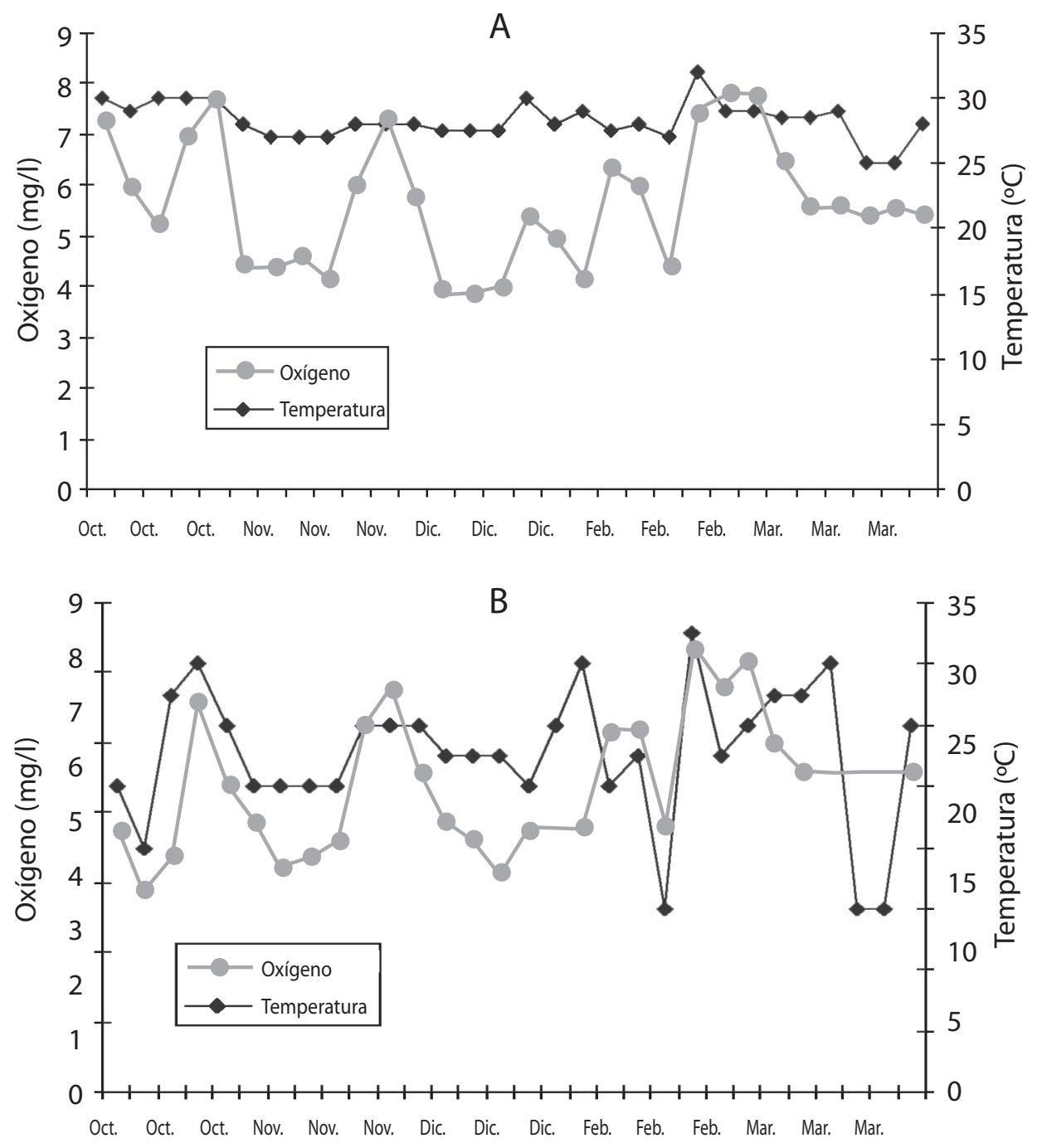

Fig. 2. Variación temporal por profundidad de la concentración de oxígeno disuelto y la temperatura para el período de octubre 1998 a marzo 1999 en el estero Punta Morales, Puntarenas, Costa Rica: a) en superficie; b) a tres metros. 


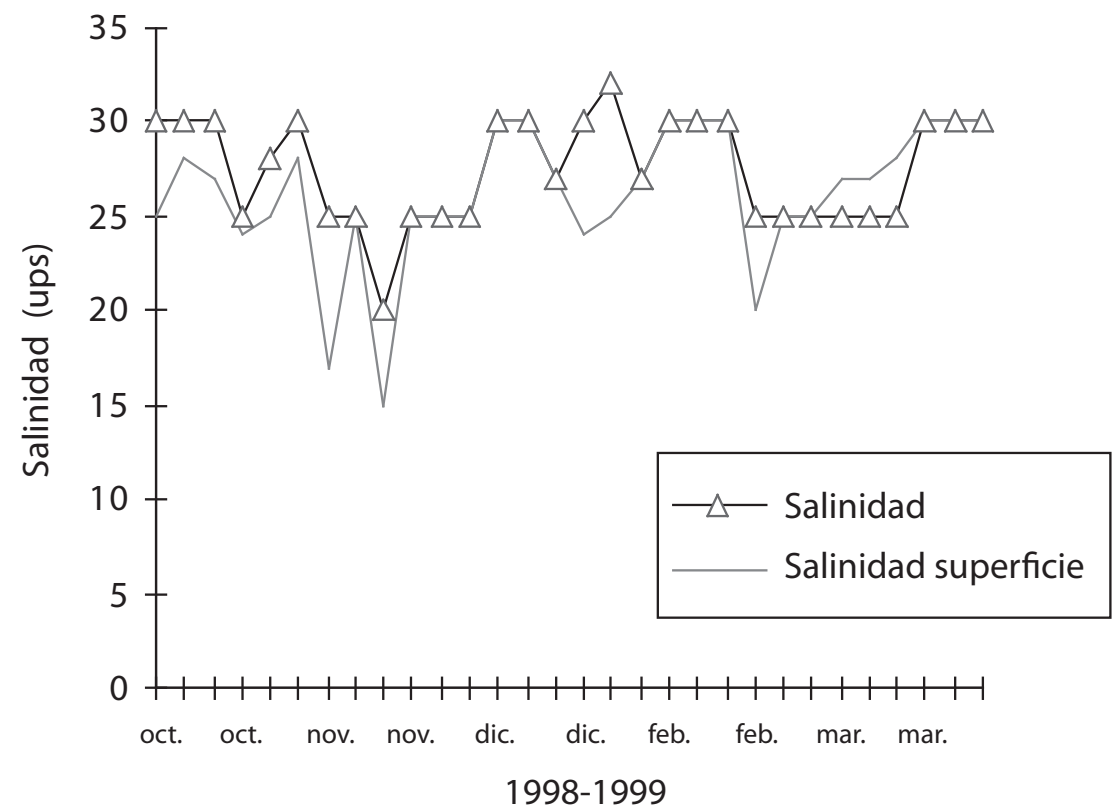

Fig. 3. Variación temporal de la salinidad por profundidad en los sitios estudiados, Golfo de Nicoya.

CUADRO 2

Variación de la profundidad del disco de Secchi (m) en los sitios estudiados

TABLE 2

Variation in Secchi disk depth (m), per site

$\begin{array}{lccc}\text { Parámetros Estadísticos } & \text { Sitio I } & \text { Sitio II } & \text { Sitio III } \\ \mathrm{N} & 10 & 10 & 10 \\ \text { Mínimo } & 0.60 & 0.39 & 0.28 \\ \text { Máximo } & 2.15 & 1.45 & 0.95 \\ \text { Media } & 1.14 & 0.85 & 0.63 \\ \text { Desviación Estandart } & 0.53 & 0.33 & 0.20\end{array}$

no presentaron variación significativa entre estaciones. No obstante, fue posible observar mínimos en ambas profundidades durante diciembre y máximos en febrero (Fig. 2 a y b). La variación espacial del oxígeno fue significativa tanto para el efecto de la marea como para la profundidad $(\mathrm{p}<0.05)$; más no así para los sitios estudiados.

Los valores de penetración de luz presentaron valores que oscilaron entre los $0.39 \mathrm{~cm}$ (sitio 1, diciembre) y $215 \mathrm{~cm}$ (sitio 2, marzo) (Cuadro 2). Los valores más altos de penetración de luz se registraron en el sitio más alejado de la costa; mientras que los mínimos se registraron en el más cercano.

Variación espacial y temporal de larvas de $P$. armatus y otros decápodos: Aunque no se encontró diferencias significativas entre estaciones $(p>0.05)$ es posible observar una mayor 
CUADRO 3

Variación por estación de los parámetros estadísticos para la densidad de larvas de Petrolisthes y de decápodos, ind/ $\mathrm{m}^{3}$

TABLE 3

Station variation of statistical parameters for the density of larvae of Petrolisthes and other decapodos, ind $/ \mathrm{m}^{3}$

$\begin{array}{llcr}\text { Parámetros Estadísticos } & \begin{array}{c}\text { Densidad de } \\ \text { Petrolisthes sp. }\end{array} & \begin{array}{r}\text { Densidad larv } \\ \text { de decápodo }\end{array} \\ \text { Estación Seca } & \text { Minimo } & 0.0 & 6.74 \\ \text { Máximo } & 6.64 & 830.81 \\ \text { Media } & 1.26 & 112.39 \\ \text { Estación lluviosa } & \text { Varianza } & 3.10 & 25772.7 \\ & \text { Desviación Estándar } & 1.76 & 160.53 \\ & \text { Mínimo } & & \\ & \text { Máximo } & 0.0 & 0.0 \\ & \text { Media } & 7.28 & 367.80 \\ & \text { Varianza } & 1.16 & 78.96 \\ & \text { Desviación Estándar } & 2.67 & 8495.76 \\ & & 1.63 & 92.17\end{array}$

densidad tanto de $P$. armatus como de larvas de decápodos en la estación seca (Cuadro 3) (Fig. $4 \mathrm{~b}$ y $5 \mathrm{~b}$ ). También los valores más altos por fecha se encontraron en la estación seca en diciembre para decápodos y en febrero para Petrolisthes (Fig. 5b y 4b). No se cuantificaron individuos en estadios de desarrollo avanzados y los recolectados pertenecían a los estadios de prezoea y zoea I.

En cuanto a la variación espacial no se encontró diferencias significativas por profundidad, ni para larvas de $P$. armatus ni para larvas de decápodos asociados $(\mathrm{p}>0.05)$. No obstante, sí se observó diferencias significativas entre mareas para larvas de P. armatus, más no así para larvas de decápodos. En el caso de Petrolisthes los valores de densidad más altos se encontraron durante la marea baja (Fig. 4a). No obstante, en el sitio 2 se observó una mayor densidad de larvas en marea alta (Fig. 6). Entre sitios sólo se observaron diferencias significativas para las larvas de decápodos (Fig. 5a).

Tiempo de desarrollo larval y factores que afectan el desarrollo: Los resultados indi- can que las hembras de $P$. armatus liberan un promedio de $160 \pm 176.7$ larvas en cada ciclo reproductivo. La mortalidad fue más intensa en los primeros tres días durante las fases de prezoea y zoea I. Debido a la alta mortalidad que se obtuvo en las fases iniciales, solo en dos oportunidades se llego al estadio de megalopa. El tiempo total promedio para llegar al estadio de megalopa fue de 19 dias.

En cuanto al comportamiento de la larva se observó en un $100 \%$ de los casos las prezoeas y zoeas I en la superficie. La zoea II no mostró preferencia por profundidad y en el caso de las megalopas sí se observó que una vez llegado a este estadio la zoea II se fue hacia el fondo y mudó en un período de 24 horas a megalopa.

Para el efecto de la salinidad se observó que en hembras a 32 ups el desove fue prácticamente inmediato a las 24 horas; mientras que las que se encontraban a 15 ups tardaron aproximadamente 72 horas. No obstante, las mortalidades fueron mayores en salinidades altas aunque estas no resultaron significativas. De las cuatro repeticiones hechas para la salinidad de 32 ups sólo en una se completó 


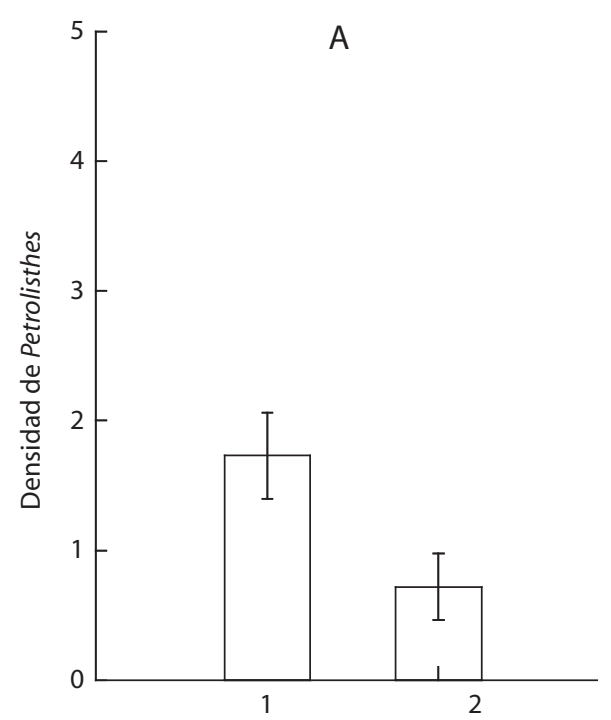

Marea baja (1) Marea alta (2)

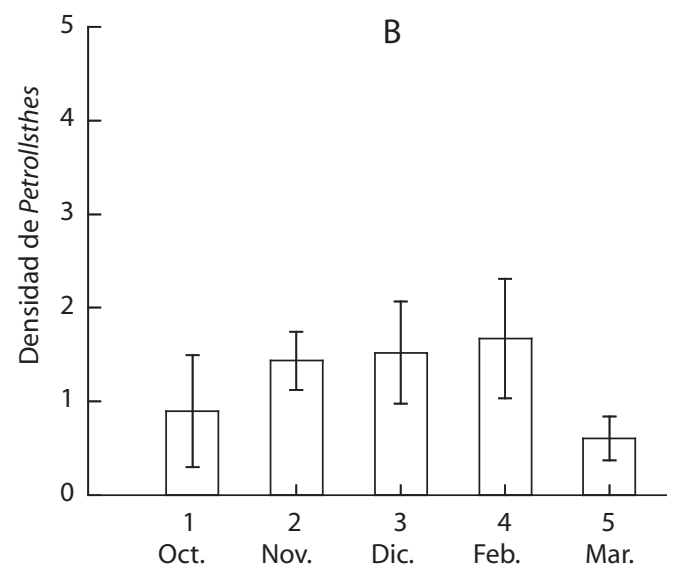

Fig. 4. Variación mareal (A) y temporal (B) de la densidad de Petrolisthes en ind $/ \mathrm{m}^{3}$. Golfo de Nicoya, 1995-1999.
A

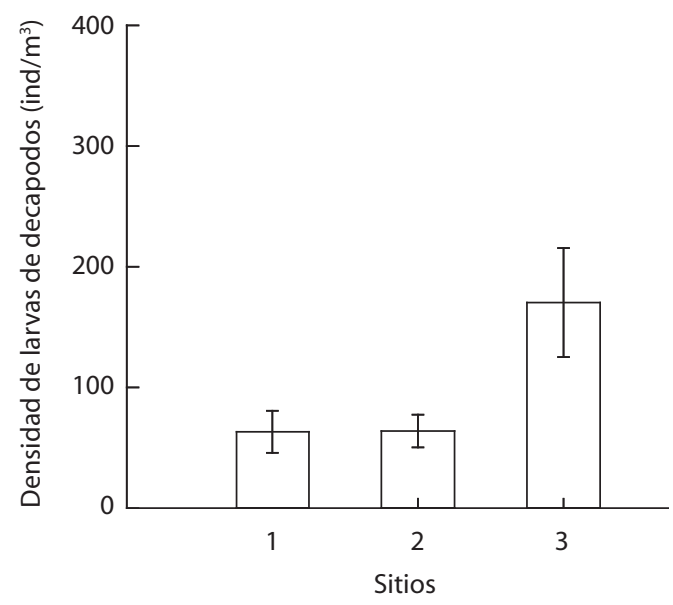

B

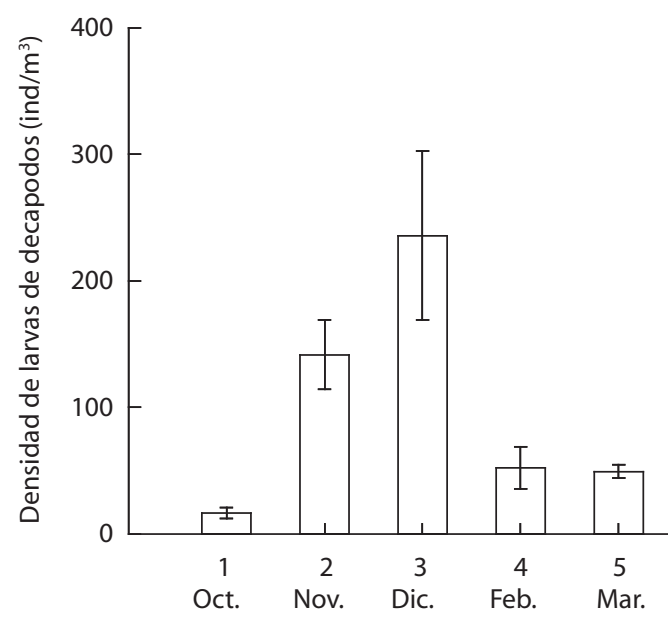

Fig. 5. Variación de la densidad de larvas de decápodos (ind $/ \mathrm{m}^{3}$ ) por sitio (A) y fecha (B). Golfo de Nicoya, 1951999. 


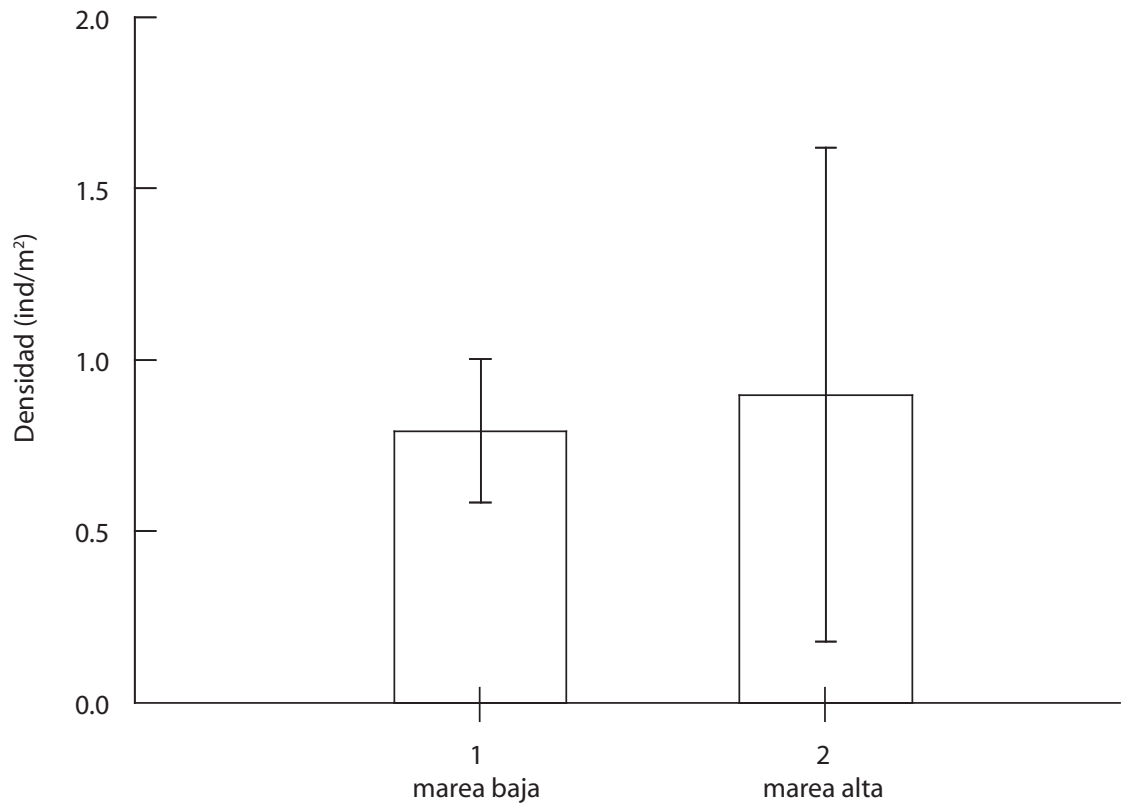

Fig. 6. Variación de la densidad larval en el sitio 2 (ubicado a 2 km de la costa).

el estadio de zoea II. Las otras tres réplicas no superaron el quinto día de desarrollo, mostrando un $100 \%$ de mortalidad al sexto día. El promedio de supervivencia a zoea II para esta salinidad fue de $12.5 \%$. Para las repeticiones realizadas a salinidades de 15 ups el promedio de supervivencia fue de $52 \%$. Al realizar la prueba de u-Mann Whitney entre las salinidades estudiadas no se encontraron diferencias significativas $(\mathrm{p}>0.05)$.

\section{DISCUSION}

Variación temporal y espacial de los parámetros ambientales: El Golfo de Nicoya es un estuario verticalmente mezclado con ciertos periodos de estratificación, causados principalmente por el aumento en la precipitación. Los valores de salinidad y temperatura suelen ser mayores en la estación seca y marea alta, que en la lluviosa y en marea baja (Voohris et al 1983, Lizano y Vargas 1983). Aunque, estacionalmente no se observaron diferencias significativas para la temperatura y el oxíge- no disuelto. Para la salinidad se observaron diferencias con máximos en la estación seca durante diciembre a tres metros y en superficie en diciembre y febrero. Los mínimos de salinidad para ambas profundidades se registraron en noviembre. La disminución en la salinidad en la estación lluviosa es causada por el aumento en precipitación a finales de octubre y principios de noviembre (Fig. 3). En estación seca la alta evaporación y la disminución en el aporte de aguas continentales eleva los valores de salinidad (Voohris et al. 1983). Entre profundidades, para este parámetro, no se observaron diferencias significativas. Esto indica que no hay estratificación y corrobora que esta zona del estuario se encuentra verticalmente mezclada (Brugnoli 1998, Córdoba 1998).

Por otra parte, se observan diferencias significativas para el oxígeno y la temperatura por profundidad. Los resultados muestran valores superiores en la superficie para la temperatura, lo cual hace cumplir con la tendencia normal de variación vertical, para este parámetro. Debido a la poca profundidad existente en la zona 
interna del Golfo no es posible la identificación de una termoclina. En cuanto al oxígeno los resultados cumplen con la ley de la solubilidad de los gases los cuales se incrementan con la disminución en la temperatura y el aumento en presión (Pine 1998, Pickard 1990). Por otro lado, en superficie existe un mayor consumo de oxígeno, ya por que las densidades de organismos son mayores y se da un mayor consumo de este gas.

Marealmente, los parámetros estudiados no variaron significativamente salvo en el caso del oxígeno disuelto. Es probable que un mayor oleaje en marea alta incremente los valores de oxígeno durante la pleamar (Pine 1998).

Para la penetración de luz los mayores valores se observan en zonas alejadas de la costa (Fig. 4). En zonas cercanas a la costa la turbidez es alta lo que impide que la luz penetre a mayor profundidad (Pine 1998). En áreas con alta penetración de luz se incrementa la zona eufótica y la capa de mezcla; lo que representa una ventaja para los organismos del zooplancton que cuentan con una mayor área de actividad y de disponibilidad de células fitopláncticas.

Distribución espacio-temporal de la larva de Petrolisthes armatus y otros decápodos asociados: Los resultados indican una mayor concentración de larvas de Petrolisthes spp. durante la estación seca; sin embargo, las diferencias entre estaciones no fueron significativas (Fig. 4a y b). Para las larvas de braquiuros, en el golfo de Nicoya, se ha encontrado variación estacional y es posible relacionar altas concentraciones de larvas de algunos géneros, no sólo en la estación seca sino también en la lluviosa (Epifanio y Dittel 1984, Dittel y Epifanio 1990). Por ello, se infiere que la mayor concentración de larvas obtenidas en la estación seca se debe a que en esta estación se presentaron salinidades intermedias entre los 28 y 30 ups, que en conjunto con otros factores brindan mayor viabilidad a la larva. Para otras especies de decápodos se ha comprobado que existe una mayor supervivencia de las larvas entre los 25 y 30 ups de salinidad (Dittel y Epifanio 1984, Messerknecht et al. 1991, Anger 1996, Anger et al. 1998).

Es importante señalar, que en algunos estadios de desarrollo la larva es más sensible a los extremos de salinidad confirmando que la capacidad de osmorregulación existe en todas las etapas de desarrollo pero es mayor en algunos estadios (Anger et al. 1996, Charmantier et al. 1998). En el ensayo experimental realizado en el presente estudio se ha observado que tanto en salinidades de 32 psu como las de 15 psu se logra completar el estadio de zoea II. No obstante, el porcentaje de supervivencia es mayor en las salinidades menores. Esto difiere de los resultados en otros estudios realizados para otros porcelánidos (Saelzer et al. 1986, Wehrtmann et al. 1996) los cuales indican que a pesar de la capacidad osmorreguladora demostrada por la larva, las salinidades altas son una limitante para el desarrollo larval de $P$. armatus. Considerando que existe un mayor éxito reproductivo en salinidades altas y una disminución en el tiempo de liberación de las larvas en salinidades elevadas; es posible que la preferencia de la larva en estadios iniciales de desarrollo por salinidades bajas obedezca a mecanismos de retención dentro del estero.

El mayor número de larvas de $P$. armatus en estación seca está relacionado, a la mayor disponibilidad de alimento y temperatura óptima que se alcanza durante esta estación las cuales aceleran y dan viabilidad al proceso de desarrollo. Para la zona interna del Golfo de Nicoya se han encontrado que la productividad primaria es mayor en la estación seca (Chaves y Birkicht 1996, Córdoba 1998). Por tanto, las variaciones en densidad de esta especie tanto en el bentos como en el plancton están reguladas por las variaciones en la productividad primaria y en el caso de la larva las variaciones en la densidad dependen en parte del aporte de progenie por parte de las poblaciones de adultos. Es posible que los meses que mostraron mayor densidad de larvas correspondan a meses en que la reproducción fue intensa y aleatoriamente se dio al unísono. Christy (1986) asegura que para $P$. armatus existe una asincronía en la liberación de larvas por las hembras ovígeras lo cual asegura 
el reclutamiento continuo. Este patrón de reclutamiento continuo es reportado para poblaciones de adultos de esta especie en el Golfo de Nicoya (Díaz-Ferguson y Vargas 2002).

La poca densidad de larvas observada en marzo, no obstante la existencia de condiciones ambientales favorables, es una evidencia de que en esta fecha la mayoría de las larvas están en fases previas a la muda de megalopa cerca del litoral; lo que impide su muestreo en plancton. La cuantificación de una mayor densidad larval durante febrero muestra que en esta fecha hubo una mayor liberación de prezoeas $\mathrm{y}$ al existir las condiciones ambientales favorables (estación seca) (Fig. 2) las densidades cuantificadas son altas.

Cabe destacar que solo se cuantificaron organismos en las fases de prezoea y zoea I y no fueron recolectados individuos en fase de zoea II, la cual es previa a la fase asentamiento "megalopa" en sitios cercanos del litoral. En estudios realizados por Dittel y Epifanio (1990) se obtuvieron resultados similares donde la mayoría de los individuos recolectados fueron zoeas I. En la presente investigación este hecho puede ser adjudicado a que los muestreos fueron matutinos y a la posible evasión de la red por parte de los organismos de mayor tamaño.

Según Christy (1986) la liberación de zoeas en $P$. armatus se da en los máximos de marea una vez esta empieza a bajar en horas cercanas al amanecer; mientras que Dittel y Epifanio (1990) encontraron mayores densidades de larvas para la familia porcelanidae durante la noche.

En cuanto a la fluctuación de la densidad de larvas de decápodos, esta mostró máximos durante diciembre y mínimos en octubre. Para los decápodos se ha señalado que tanto la temperatura como la salinidad afectan su desarrollo larval (Anger et al. 1994). Es probable que los máximos en densidad obedezcan a condiciones óptimas en la productividad primaria y en los factores anteriormente mencionados. En estudios efectuados en poslarvas de la familia Penaeidae también se registraron en la parte interna del Golfo de Nicoya las abundancias máximas durante diciembre (Kitami 1996).
Esto indica, tal como se infirió anteriormente, que en este mes las condiciones ambientales en el medio pelágico son óptimas. En cuanto a la baja densidad de larvas registradas en estación lluviosa se debe principalmente a las condiciones ambientales, que aunque están en el ámbito de tolerancia de la larva, no son las óptimas para la reproducción en el bentos lo que disminuye el aporte de larvas del plancton al bentos. Este hecho fue demostrado en los ensayos de liberación de larvas con hembras de P. armatus y para otras especies de decápodos que habitan la zona se ha encontrado una asociación entre éxito reproductivo y altas salinidades (Dittel et al. 1985, de Vries 1983).

Variación espacial: La distribución espacial de la larva de $P$. armatus muestra que el principal factor que regula la distribución es la marea, ya que se encuentraon diferencias significativas entre la bajamar y la pleamar. Smith y Stoner (1993) indican que en el proceso de transporte neto de las larvas los factores que influyen principalmente son el estrés del viento y la oscilación mareal. Aunque otros como la intensidad de las corrientes costeras, variaciones en la dirección del viento debidas a la precipitación, tipo de circulación en la zona, advección, difusión y condiciones físico-químicas juegan un papel importante en el transporte larval (Shanks 1985-87, McGurk 1989, Hill 1991, McConnaughey et al. 199295, Anger et al. 1994). En otros estudios sobre retención y dispersión larval realizados en este estuario se destacan diferencias significativas causadas por la variación en el flujo mareal (Wehrtmann y Dittel 1990).

En el presente estudio se registraron mayores densidades en la marea baja, lo cual difiere con el estudio realizado por Wehrtmann y Dittel (1990) (Fig. 5a). Esto se debe a que en el estudio citado anteriormente se midió la variación de especies de larvas que utilizaban las hojas de mangle como transporte y se cuantificaron larvas de todos los estadios de desarrollo, incluyendo las previas al asentamiento. Es importante señalar que los mecanismos de retención son muy variados 
entre especies y mientras unas especies transportan sus larvas hacia fuera del estuario; en otras el mecanismo de transporte es contrario (Dittel et al. 1982, Epifanio et al. 1989, Dittel y Epifanio 1990, Dittel et al. 1991, Smith y Stoner 1993, Chen et al. 1997). Esto hecho se demuestra al no registrarse diferencias significativas para larvas de decápodos entre marea, ya que en algunos casos la abundancia de un determinado grupo con un tipo específico de mecanismo de retención altera los resultados del total de larvas estudiadas.

Para $P$. armatus se propone que existe exportación de larvas en etapas iniciales a través de la marea baja, mientras que en etapas posteriores de desarrollo la larva entra al estero (Dittel et al. 1991). En base a esta premisa y a los resultados se puede inferir que $P$. armatus libera sus larvas en el pico máximo de marea alta y luego estas son arrastradas hacia fuera en la baja mar. Este hecho concuerda con lo encontrado por Cristy (1986) en el Pacífico panameño donde las hembras de $P$. armatus liberan de larvas durante el máximo de marea. Por otro lado, las oscilaciones observadas en la densidad larval de esta especie para las fases de prezoea y zoea I obedecen a la existencia de un mecanismo de transporte mareal y por ello su mayor abundancia en marea baja. Estos resultados son concordantes con los obtenidos por Dittel y Epifanio (1990) quienes encontraron una mayor densidad en la marea baja para los estadios iniciales y en marea alta para fases avanzadas como la zoea II y la megalopa.

Entre los sitios estudiados no se registraron diferencias significativas para la densidad de larvas ni para los parámetros físico-químicos. Los sitios 1 y 3 que se encontraban muy cerca de la costa rocosa mostraron la mayor densidad de larvas. En el sitio 2 que se encontraba un tanto alejada de la costa sí se observó un mayor número de larvas de Petrolisthes en marea alta con lo cual se plantea una entrada de las larvas hacia la costa a través de la marea alta y una salida una vez entra empieza a bajar (Fig. 6). Para larvas de otros decápodos sí se observaron diferencias significativas por sitio, con una mayor densidad en el sitio 3 (Fig. 5a). Este sitio está ubicado en la zona interna del estero de Punta Morales; donde existe un mayor aporte de larvas de especies que habitan el manglar, así como de otras que habitan aguas más oceánicas lo que indica que esta zona es importante en el criadero y reproducción de los decápodos (Jesse 1996).

En cuanto a la influencia de la penetración de luz, no existió una relación directa entre este parámetro y la abundancia de larvas. Otros estudios muestran que el transporte de la larva responde dos veces más al flujo mareal que a la intensidad de luz (Forward 1988). Para larvas completamente fitoplanctívoras se esperaría una relación directa debido a la abundancia de células fitopláncticas en zonas de mayor penetración de luz. Sin embargo, nuestros resultados muestran las altas densidades de larvas asociadas a los sitios cercanos a la costa donde los valores de penetración fueron menores. Debido a que las larvas de Petrolisthes sp. son omnívoras es posible que estas muestren preferencia hacia zonas donde algunos grupos del zooplancton que son sus presas son abundantes.

Al no observarse variación vertical significativa en la densidad a pesar de que se registraron más larvas en profundidades de 3 metros, se demuestra que esta especie presenta un ámbito considerable para tolerar variaciones en parámetros como el oxígeno y la temperatura que presentaron variación significativa en las dos profundidades estudiadas (Fig. 2a y b). Por otro lado, la existencia de un mayor número de larvas cerca del fondo apoya el paradigma de que las larvas de decápodos prefieren las profundidades en donde la intensidad de corriente es menor (Hill 1991, Smith y Stoner 1993).

\section{AGRADECIMIENTOS}

Este estudio es parte del segundo capítulo de la Tesis de Maestría presentada por el primer autor en el Sistema de Estudios de Posgrado de la Universidad de Costa Rica. Los autores agracecen al personal administrativo y científico del Centro de Investigación en Ciencias del Mar y Limnología (CIMAR) de 
la Universidad de Costa Rica y al personal de la ECMAR (Punta Morales) por el apoyo logístico brindado para la realización de esta investigación. Asimismo, a la Organización de Estados Americanos (O.E.A) por proveer al primer autor de una beca de investigación para la realización de estudios de Maestría en la Universidad de Costa Rica.

\section{RESUMEN}

Durante octubre y noviembre de 1998 (estación lluviosa) y diciembre, febrero y marzo de 1999 (estación seca) se muestreó la distribución de la larva de Petrolisthes spp, así como la densidad del total de las larvas de decápodos en superficie y a 3 metros de profundidad. Los muestreos se realizaron en tres sitios de la zona de Punta Morales, Golfo de Nicoya, Costa Rica, por medio de arrastres con una red de plancton de $280 \mu \mathrm{m}$. Se recolectaron un total de 6014 larvas de decápodos de las cuales $73(1.21 \%)$ pertenecían al género Petrolisthes spp. No se encontraron diferencias significativas entre estaciones $(p>0.05)$, sin embargo fue posible observar una mayor densidad tanto de P. armatus como de larvas de decápodos en estación seca. Temporalmente el único parámetro físico-químico que varió significativamente fue la salinidad. En cuanto a la variación espacial no se encontraron diferencias significativas por profundidad, ni para Petrolisthes spp. ni para larvas de decápodos asociados ( $\mathrm{p}>0.05)$, a pesar de que la variación de algunos parámetros como la temperatura y el oxígeno si lo fue. Entre mareas se encontraron diferencias significativas para las larvas de Petrolisthes spp., no así para larvas de decápodos. Para la especie estudiada se observó un mayor número de larvas en marea baja. Entre los sitios hubo diferencias significativas para las larvas de decápodos, pero no para Petrolisthes spp.; físico-químicamente estos sitios son muy parecidos y solo difieren en penetración de luz, lo que parece no afectar a la larva de Petrolisthes. spp. Los resultados demuestran que el principal factor que regula la concentración temporal de las larvas es la salinidad, mientras que espacialmente las oscilaciones en el flujo mareal son de mayor importancia. Debido a las diferencias observadas marealmente para la concentración de larvas se propone el siguiente mecanismo: P. armatus libera sus larvas en el máximo de marea, éstas salen hacia fuera de la costa con la marea baja y se reincorporan con la marea alta, cuando están cerca de la fase previa al asentamiento (la megalopa). Adicional a los resultados en el medio natural para esta especie también se estudió en el laboratorio la duración del periodo larval y la supervivencia de las larvas a salinidades extremas utilizándose los valores máximos y mínimos registrados en el medio natural (15 ups y 35 ups). Este ensayo demostró que las larvas presentan un mayor porcentaje de supervivencia en salinidades menores (15 ups) y que la duración del período de desarrollo es de 19 dias.

Palabras claves: Crustacea, Porcellanidae, Petrolisthes, larva, dinámica de poblaciones, Golfo de Nicoya, Costa Rica.

\section{REFERENCIAS}

Anger, A., E. Spivak, C. Bas, D. Ismael \& T. Luppi. 1994. Hatching rhythms and dispersion of decapod crustacean larvae in a brackish coastal lagoon in Argentina. Helgolander Meeresun. 48: 445-466.

Anger, K. 1996. Salinity tolerance of the larvae and first juveniles of a semiterrestial grapsid crab, Armases miersii (Rathbun). J. Exp. Mar Biol. Ecol. 202: 205-223.

Anger, K., E. Spivak \& T. Luppi. 1998. Effects of reduced salinities on development and bioenergetics of early larval shore crab, Carcinus maenas. J Exp Mar Biol Ecol. 220: 287-304.

Brugnoli, E. 1998. Factores abióticos y bióticos que regulan la estructura y dinámica de la comunidad fitoplánctica en la zona de Punta Morales, Golfo de Nicoya. Tesis de Maestría. Universidad de Costa Rica, Ciudad Universitaria Rodrigo Facio, San Pedro, San José, Costa Rica. 134p.

Córdoba, R. 1998. Primary productivity in the water column of Estero Morales, a mangrove system in the Gulf of Nicoya, Costa Rica. Rev. Biol. Trop. 46: 257-262.

Charmantier, G., M. Charmantier-Daures, K. Anger. 1998. Ontogeny of osmoregulation in the grapsid crab Armases miersii (Crustacea: Decapoda). Mar. EcolProg. Ser. 162: 285-292.

Chaves, J. \& M. Birkicht. 1996. Equatorial Subsurface Water and the nutrient seasonality distribution of the Gulf of Nicoya, Costa Rica. Rev. Biol. Trop. 44 (Suppl. 3): 42-47.

Chen, Y.H., P.T. Shaw \& T.G. Wolcott. 1997. Enhancing estuarine retention of planktonic larvae by tidal currents. Estuar. Coast. Shelf. S. 45: 525-533.

Christy, J. 1986. Timing of larval release by intertidal crabs on an exposed shore. B. Mar. Sci. 39: 176-191.

De Vries. M.C., C.E. Epifanio \& A.I. Dittel. 1983. Reproductive periodicity of the Tropical crab Callinectes arcuatus Orway in Central America. Estuar. Coast. Shelf. S. 17: 709-715.

Díaz-Ferguson, E. \& J.A. Vargas. 2002. Abundance of Petrolisthes armatus (Crustacea: Porcellanidae) in 
a tropical estuarine intertidal rocky beach, Gulf of Nicoya estuary, Costa Rica. Rev. Biol. Trop. 49: 97-101.

Dittel, A. \& C.E. Epifanio. 1982. Seasonal abundance and vertical distribution of crab larvae in Delaware Bay. Estuaries 5: 197-202.

Dittel, A. \& C.E. Epifanio. 1984. Growth and Development of the portunid crab Callinectes arcuatus Ordway: zoea, megalopae and juveniles. J. Crustacean Biol. 4: 491-494.

Dittel, A., C.E. Epifanio \& J.B. Chavarría. 1985. Population Biology of the portunid crab Callinectes arcuatus Orway in the Gulf of Nicoya, Costa Rica, Central America. Estuar. Coast. Shelf. S. 20: 593-602.

Dittel, A., C.E. Epifanio \& O. Lizano. 1991. Flux of crab larvae in mangrove creek in the Gulf of Nicoya, Costa Rica. Estuar. Coast. Shelf. S. 32: 129-140.

Eckman, J. 1996. Closing the larval loop: linking larval ecology to the population dynamics of marine benthic invertebrates. J. Exp. Mar. Biol. Ecol. 200: 207-237.

Epifanio, C.E., C. Valenti \& A. Pembroke. 1984. Dispersal and recruitment of blue crab larvae in Delaware Bay, U.S.A. Estuar. Coast. Shelf. S. 18: 1-12.

Epifanio, E., A. Masse \& R. Garvine. 1989. Transport of blue crab larvae by surface currents off Delaware Bay, USA. Mar. Ecol. Prog. Ser. 54: 35-41.

Forward, R. 1988. Diel vertical migration: zooplancton photobiology and behavior. Oceanogr. Mar. Biol. Annu. Rev. 26. 361-393.

Gore, R. 1972. Petrolisthes armatus (Gibbes, 1850): The development under laboratory conditions of larvae from a pacific specimen (Decapoda: Porcellanidae). Crustaceana 22: 68-83

Hill, A.E. 1991. Vertical migration on tidal currents. Mar. Ecol. Prog. Ser. 75: 39-54.

Hill, A.E. 1991. A Mechanism for horizontal zooplancton transport by vertical migration in tidal currents. Mar. Biol. 111: 485-492.

Jesse, S. 1996. Demersal crustacean assemblages along the Pacific coast of Costa Rica: a quantitative and multivariate assesment based on the Victor Hensen Costa Rica Expedition (1993/1994). Rev. Biol. Trop. 44: 115-134.

Kitami, H. 1996. Abundance and size frecuency of postlarvae of Penaeus occidentalis in the Gulf of Nicoya,
Costa Rica. 1996-1997. Rev. Biol. Trop. 44 (Suppl. 3): 437-440.

Lizano, O.G \& J.A. Vargas.1983. Distribución espaciotemporal de la salinidad y la temperatura en la parte interna del Golfo de Nicoya. Tecnología en Marcha 12: 3-16.

Messerknecht, I., M. Montu \& K1. Anger. 1991. Larval development and salinity tolerance of Brazilian Eurytium limousum (Say, 1818) (Brachyura: Xanthidae) reared in the Laboratory. Meeresforschung. 33: 275-296.

Miron, G; Bernard, B. \& E. Bourget. 1995. Use of larval supply un benthic ecology: testing correlations between larval supply and larval settlement. Mar. Ecol. Prog. Ser. 124: 301-305.

McConnaughey, R.A., D.A. Armstrong, B.M. Hickey \& D.R. Gunderson. 1992. Juvenile Dungeness crab (Cancer magister) recruitment variability and oceanic transport during the pelagic larval phase. Can. J. Fish. Aquat. Sci. 49: 2028-2044.

McConnaughey, R., D. Armstrong, B. Hickey \& D.R. Gunderson. 1994. Interannual variability in coastal Washingrton Dungeness crab (Cancer magister) populations: larval advection and the coastal landing strip. Fish. Oceanogr. 3: 22-38.

McConnaughey, R., D. Armstrong \& B. Hickey. 1995. Dungeness crab (Cancer magister) recruitment variability and Ekman Transport of larvae. Ices. J. Mar. Sci. Symp. 199: 167-174.

McEdward, L. 1995. Ecology of Marine Invertebrate Larvae. CRC Marine Science Series. Boca Raton, Florida, EEUU. 248p.

McEdward, L. 1997. Reproductive strategies of marine benthic invertebrates revisited: Facultative feeding by planktotrophic larvae. Am. Nat. 150: 48-71.

McGurk, M. 1989. Advection, diffusion and mortality of Pacific herring larvae Clupea harengus pallasi in Bamfield Inlet, British Columbia. Mar. Ecol. Prog. Ser. 51: 1-18.

Oliveira, E. \& M. Masunari. 1995. Population structure of Petrolisthes armatus (Gibbes, 1850) (Decapoda: Porcellanidae) from Farol isle, Matinhos, Brazil. Rev. Bras. Zool. 12:355-371.

Osman, R. \& R. Whitlatch. 1995. The influence of resident adults on recruitment: a comparison to settlement. J. Exp. Mar. Biol. Ecol. 190: 169-198.

Olson, R.R. 1985. The consecuences of short-distance larval dispersal in a sessile. Marine Invertebrate Ecology 66: 30-39. 
Palacios, J.A., J.A. Rodríguez \& R.A. Angulo. 1993. Estructura poblacional de Penaeus stylirostris (Decapoda: Penaeidae), en el Golfo de Nicoya, Costa Rica. Rev. Biol. Trop. 41: 233-237.

Pickard, G.L. \& W., Emery. 1982. Descriptive Physical Oceanography. Pergamon. Elmsford, Nueva York, EEUU. 249 p.

Pine, P. 1998. Invitation to Oceanography. Jones and Barlett. Sudbury, Massachusetts, EEUU. 508p.

Ray, G. 2005. Invasive marine and estuarine animals of the south Atlantic and Puerto Rico. Aquatic Nuisance Species Research Program. Aquatic Nuisance Species Research Program. Alexandria, Virginia, EEUU. 14 p.

Sastry, A.N. 1983. Pelagic larval ecology and development. In Doroty Bliss (Ed.). The Biology of Crustacea. Academic. Fairview Park, Ohio, EEUU. 319 p.

Shanks, A. 1985. Behavioral basis of internal -waveinduced shoreward transport of megalopae of the crab Pachygrapsus crassipes. Mar. Ecol. Prog. Ser. 24: $289-295$

Shanks, A \& W. Wright. 1987. Internal-wave-mediated shoreward transport of cyprids, megalopae, and gammarids and correlated longshore difference in the settling rate of intertidal barnacles. J. Exp. Mar. Biol. Ecol. 114: 1-13.

Smith, N \& W. Stoner. 1993. Computer simulation of larval transport through tidal channels: Role of Vertical Migration. Estuar. Coast. Shelf. Sci. 37: 43-58.

Vargas, J.A. 1995. The Gulf of Nicoya estuary, Costa Rica: Past, Present, and future cooperative research. Helgoländer Meeresun. 49: 821-828.

Voohris, A.D., C.E. Epifanio, D. Maurer, A. Dittel \& J.A. Vargas. 1983. The estuarine character of the Gulf of Nicoya, an embayment on the Pacific coast of Central America. Hydrobiologia. 99: 225-237.

von Wangelin, W. \& M. Wolff. 1986. Comparative biomass spectra and species composition of the zooplankton communities in Golfo Dulce and Golfo de Nicoya, Pacific coast of Costa Rica. Rev. Biol. Trop. 44 (Suppl. 3): $135-155$.

Watzin, M. 1986. Larval settlement into marine soffsediment systems: Interactions with the Meiofauna. J. Exp. Mar. Biol. Ecol. 98: 65-113.

Wehrtmann, I. \& A. Dittel. 1990. Utilization of floating mangrove leaves as a transport mechanism of estuarine organism, with emphasis on decapod Crustacea. Mar. Ecol. Prog. Ser. 60: 67-73. 
